\title{
Das TSPP-Modell - Eine Blaupause für die Coaching-Prozessforschung
}

\author{
Silvia Deplazes · Eva-Maria Graf² (D) Hansjörg Künzli
}

Online publiziert: 9. November 2018

(c) Der/die Autor(en) 2018

\section{Zusammenfassung}

Ausgangslage: Coaching begleitet Veränderungen bei KlientInnen. Diese Veränderungsprozesse sind nicht sichtbar; sichtbar ist ausschließlich der Coachingprozess - die Interaktion zwischen Coach und KlientIn. Im Prozess intervenieren Coaches sowohl in Reaktion auf die KlientInnen als auch mit Bezugnahme auf ihr Theorie- und Handlungssystem. Dies kann mit dem Begriff der appropriate responsiveness umschrieben werden.

Bestehende Coaching-Prozessforschung fokussiert entweder theoriegeleitet die Intensität und Häufigkeit von Phänomenen (psychologische Forschungstradition) oder deskriptiv die Sequenzialität des Gesprächs (linguistische Forschungstradition).

Ziel: Ziel des Beitrags ist es, eine erste Brücke zwischen normativ-theoretischen (psychologischen) und deskriptiv-phänomenologischen (linguistischen) Forschungsansätzen zu schlagen. Dazu wird ein Modell entwickelt, welches als Strukturierungshilfe der Erforschung von Coachingprozessen dienen kann. Mit dem Modell sollen Fragen nach dem „Was?“, dem „Wie?“ und dem „Wie oft?““ in Beziehung zum Verlauf des Coachings und somit dem „Wann?“, bzw. „Wo im Prozess?“, gesetzt werden können.

Methode: Die Konzeption des Modells beruht auf der Auswertung, Zusammenführung und Erweiterung existierender Modelle zu Veränderung und zu Interaktion.

Ergebnisse: Das Turn-Sequenz-Phase-Prozess-Modell (TSPP-Modell) fokussiert gleichzeitig drei Ebenen von Coachingprozessen, Turns, Sequenzen und Phasen. Es ermöglicht Prozessforschung im zeitlichen Verlauf zu strukturieren.

Schlüsselwörter Coachingprozess · Veränderungsprozess · Appropriate responsiveness · Coaching-Prozessforschung · TSPP-Modell

\section{The TSPP Model - A Blue Print for Coaching Process Research}

\section{Abstract}

State of the art: Coaching accompanies change in clients. These change processes cannot be observed; what can be observed are coaching processes, i.e. the interaction between coach and client. In a coaching process, coaches intervene both in response to clients' reactions and in response to their own theory and action logic. This can be subsumed under "appropriate responsiveness" in Stiles, Honos-Webb and Surko (1998) sense. Existing coaching process research focusses either on intensity and frequency of one or more phenomena (in the context of theory-based psychological research tradition) or focusses descriptively on the sequentiality of the coaching conversation (in the context of linguistic research tradition).

\section{Publisher's Note Springer Nature remains neutral with regard} to jurisdictional claims in published maps and institutional affiliations.

\section{Silvia Deplazes}

deze@zhaw.ch

1 Zürcher Hochschule für Angewandte Wissenschaften, Pfingstweidstrasse 96, 8005 Zürich, Schweiz

2 Alpen-Adria-Universität, Klagenfurt, Österreich 
Goal: The goal of our contribution is to build a first bridge between normative-theoretical (psychological) and descriptive-phenomenological (linguistic) approaches in coaching research. To this end, we develop a model that functions as a blue print for the analysis of coaching processes. With the help of this model, questions addressing "what", "how" and "how often" can be related to the coaching process and thus to "when" and "where along the process".

Method: The conceptualization of the model relies on analyzing, merging and expanding existing models on change and interaction.

Findings: The Turn-Sequence-Phase-Process Model (TSPP-Model) focuses concurrently on the level of turns, sequences and phases as components of coaching processes. This allows for structuring process research in its time course.

Keywords Coaching process $\cdot$ Change process $\cdot$ Appropriate responsiveness $\cdot$ Coaching process research $\cdot$ TSPP-model

\section{Einleitung}

Coaching und Beratung sind zentrale Bestandteile gesellschaftlicher und arbeitsplatzbezogener Prozesse und werden im Zuge der Individualisierung, Personalisierung und Diagnostizierung unserer Gesellschaft in naher $\mathrm{Zu}$ kunft wohl an Bedeutung zunehmen. Dieser praktischen Bedeutung und Etablierung steht eine in vielen Bereichen (immer noch) unbefriedigende wissenschaftliche Fundierung gegenüber (Fillery-Travis und Cox 2014; Passmore et al. 2013). Was man allerdings bereits gut empirisch belegen kann, ist, dass Coaching Wirkung zeigt (vgl. dazu die Metaanalysen von Burt und Talati 2017; De Meuse et al. 2009; Jones et al. 2016; Sonesh et al. 2015; Theeboom et al. 2014). Bedeutend schwieriger aufzuzeigen ist demgegenüber, wo und wie genau im Verlauf von Coachingund Beratungsprozessen Wirkung erzeugt wird (de Haan et al. 2010). Das hier entwickelte TSPP-Modell (TurnSequenz-Phase-Prozess-Modell), das der Coaching-Prozessforschung als Strukturierungshilfe dienen soll, bietet dabei eine erste Hilfestellung.

Bis dato existieren - in einer für den Zweck des Beitrags notwendigen Vereinfachung - zwei unterschiedliche empirische Perspektiven auf den Coachingprozess, bzw. auf das Geschehen im Coaching, das die Veränderung bei KlientInnen unterstützt: Die psychologische Prozessforschung untersucht, welche Wirkvariablen dem Coaching zugrunde liegen; der Fokus liegt auf dem „Was“ und dem „Wie oft“ (z.B. Greif 2008). Die linguistische Coaching-Prozessforschung zeigt den sequentiellen Verlauf der sozialen Interaktionen Coaching auf und beschriebt diesen detailliert; der Fokus liegt auf dem „Wie“ und dem „Wann“ im Kontext der lokalen Wirksamkeit kommunikativer Praktiken (z. B. Graf 2015, 2019; Graf und Jautz 2019).

Während Konversationsanalyse und Gesprächsanalyse die Sequenzialität der Gespräche thematisieren, dabei allerdings den Kontext, also die konkreten Umstände eines Coachings unberücksichtigt lassen, konzentriert sich die psychologische Prozessforschung auf (Wirk-)Faktoren des Gespräches, ohne diese in den Kontext des Gesprächsablaufes einzubetten. Beide, sowohl die deskriptiv- phänomenologisch als auch die normativ-theoretische Herangehensweise stossen bei der Analyse der Interaktion an ihre Grenzen (vgl. z.B. Deppermann 2018). Durch den hier gewählten disziplinenübergreifenden Zugang sollen die Zusammenführung der beiden Perspektiven unterstützt und Forschungsdesiderate in der linguistischen und psychologischen Prozessforschung bedient werden.

Ziel des vorliegenden Beitrags ist es, ein Modell zu entwickeln, das der Coaching-Prozessforschung eine Strukturierungshilfe bietet, um Fragen nach dem „Was?“, dem „Wie?“ und dem „Wie oft?“ von Phänomenen in Beziehung zu „Wann?““, bzw. „Wo im Prozess?““, setzen zu können. Das hier vorgeschlagene Modell soll helfen, einen vollständigeren Blick auf Coachingprozesse zu erlangen. Das TSPPModell (Turn-Sequenz-Phase-Prozess-Modell) nimmt dabei den Grundgedanken der appropriate responsiveness nach Stiles (Stiles et al. 1998) auf, welcher die doppelte Bezugnahme (einerseits auf die KlientInnen, andererseits auf die Theorie, hier allerdings sehr eingeschränkt auf Phasenmodelle) propagiert. Damit wird einer zentralen empirischen Herausforderung der Prozess-Wirkungsforschung begegnet.

Die Modell-Entwicklung basiert auf Wissen zur Interaktion im Coaching, vor allem formalen Elementen der Gesprächsstrukturierung (Turn, Sequenz, Phase und Prozess) sowie empirischem Wissen zur Veränderung von Verhalten. Die Konzeption des Modells beruht dabei auf der Auswertung, Zusammenführung und Erweiterung existierender Modelle. $\mathrm{Zu}$ diesem Zweck werden einleitend die Ebenen der Prozessforschung und die Sequenzialität von Interaktion reflektiert. Es folgt eine Auseinandersetzung mit Veränderungsmodellen und der Responsivität nach Stiles et al. (1998). Der Stand der Coaching-Prozessforschung sowie eine Übersicht über Phasenmodelle runden die Darstellung der Grundlagen ab. Auf dieser Basis wird in einem zweiten Teil das TSPP-Modell konzipiert und abschliessend diskutiert. 


\section{Grundlagen für die Modell-Entwicklung}

\subsection{Ebenen der Prozessforschung und Fokus des vorliegenden Modells}

Für die Prozess-Ergebnisforschung in der Psychotherapie schlagen Orlinsky et al. (2004) neun Analyseebenen vor. Ordnungskriterium ist die Zeitdauer des interessierenden Geschehens, beginnend bei Sekundenbruchteilen (Veränderung der Blickrichtung, Mikrobewegungen der Gesichtsmuskulatur) auf der ersten Ebene bis hin zur Betrachtung der individuellen, therapeutischen Karriere über die Lebensspanne auf der neunten Ebene. Der Fokus der Forschung ist laut Orlinsky et al. (2004) letztlich immer eine forschungsparadigmatische Frage.

Hier wird ein Dreiebenen-Ansatz mit einer Unterteilung in Mikro-, Meso- und Makroebene der Kommunikation im Coaching vorgeschlagen. Diese Unterteilung ist rein deskriptiv und soll der groben Orientierung dienen. Untersuchungen auf der Mikro-Ebene können sich auf die Synchronisierung der Spiegelneuronen oder die immer noch feingliederig organisierte Synchronisierung der nonverbalen Kommunikation beziehen (Ramseyer und Tschacher 2011, 2014). Auf der Meso-Ebene verorten wir die direkten, ohne technische Hilfsmittel beobachtbaren Realisierungen der Interaktionen im Coaching. Diese umfassen verbale und non-verbale Elemente wie z.B. Nicken, Interjektionen, einzelne Redebeiträge, Gesprächssequenzen bis hin zur Betrachtung mehrerer Sitzungen. Der Makro-Ebene ordnen wir, ähnlich wie im Mehrebenen-Modell von Greif (2008), Team-, Organisations-, Landeskultur oder Theorien und Haltungen von Coaches zu.

Ein Modell, das der Coaching-Prozessforschung dienen soll, kann zwar vom theoretischen Anspruch her versuchen, alle Ebenen des Geschehens abzubilden, eine gleichzeitige Untersuchung aller Ebenen scheint aber in der konkreten Umsetzung unmöglich. Eine Lösung liegt darin, die Reichweite des Modells zu begrenzen, so dass es den Möglichkeiten der Forschung entspricht. Dies ist der Weg, den wir hier wählen wollen. Der Fokus liegt auf der sprachlichen Interaktion. Damit klärt sich gleichzeitig, was unter einem Coachingprozess verstanden wird. Als Coachingprozess wird die Interaktion zwischen Coach und KlientIn verstanden, sei dies im Rahmen von face-to-face Coaching oder Online Coaching. Elemente ausserhalb der direkten und damit beobachtbaren Interaktion, wie z. B. Hausaufgaben, die KlientInnen zwischen den Sitzungen erarbeiten oder mentale Erkenntnisprozesse, werden im vorliegenden Modell nicht abgebildet. Der Anfangs- und der Endpunkt eines so definierten Coachingprozesses lassen sich bestimmen. Ein Coachingprozess beginnt mit dem ersten Kontakt zu Beginn der ersten Sitzung und endet mit der gemeinsamen Feststellung, dass das Coaching nun beendet ist und kein neuer Termin mehr vereinbart wird, d.h. mit der Gesprächsbeendigung der letzten Sitzung.

\subsection{Die Sequenzialität sozialer Interaktion}

Konversationsanalyse und linguistische Gesprächsanalyse haben als Grundannahme, dass soziale Interaktionen wie Alltagsgespräche oder - wie im vorliegenden Fall Beratungsgespräche - sequentiell aufgebaut sind (Deppermann 2008; Schegloff 2007). Ein Gesprächsbeitrag (Turn) nimmt interaktiv immer Bezug auf den vorangehenden und bildet die Voraussetzung für den nachfolgenden Turn. Ein Gespräch ist somit eine Abfolge von mehr oder weniger zueinander passenden und kontextangepassten Gesprächsbeiträgen. Mehrere Turns, die aufgrund der konditionalen Relevanz oder bedingten Erwartbarkeit (Henne und Rehbock 2001) sowohl thematisch als auch gesprächsorganisatorisch verbunden sind, bilden aus dieser Perspektive eine Sequenz. Soziale Interaktionen basieren auf diesen Handlungssequenzen.

\subsection{Veränderungsmodelle}

Ziel eines jeden Coaching ist eine Veränderung für KlientInnen. Um mit Whitworth et al. (1998, S. xix) zu sprechen, ,(p)eople come to coaching for lots of different reasons, but the bottom line is change“. Veränderung kann dabei die Erweiterung oder Umstrukturierung der Deutungsmuster oder Handlungsoptionen der KlientInnen umfassen (vgl. z.B. Schreyögg 2012). Im Hinblick auf Veränderungsprozesse stehen verschiedene empirisch überprüfte Modelle zur Verfügung, welche alle beschreiben, wie es von der Wahrnehmung eines problematischen Verhaltens, resp. dem Wunsch einer Handlung, zur Umsetzung und Bewertung sowie Aufrechterhaltung derselben kommt. In diesen Modellen wird stets das Konzept der Phase verwendet. In der Gesundheitspsychologie sind das HAPA Modell (Schwarzer 2008) sowie das Transtheoretische Modell (Prochaska et al. 1991), in der Motivationspsychologie ist das Rubikon-Modell zu nennen (Heckhausen und Heckhausen 2010). Exemplarisch sei hier das Rubikon-Modell vorgestellt, da es Eingang in einschlägige Coaching-Literatur gefunden hat (z.B. Greif und Benning-Rohnke 2015). Es beschreibt den Handlungsverlauf von den Wünschen einer Person bis zur Bewertung des erreichten Handlungsziels in vier (Achtziger und Gollwitzer 2010), bzw. in Anlehnung an Grawe (1998), in fünf Phasen (Storch und Krause 2007): Das Bedürfnis zeigt eine Diskrepanz zwischen Soll- und Istwert auf (Phase 1), welche es sorgfältig zu explorieren gilt. Entwickelt sich aus dem Bedürfnis ein Motiv (Phase 2), besteht ein bewusstes Ziel. Es folgen das Überschreiten des „Flusses“ und damit der Schritt zur Intention (Phase 3), d. h. es besteht jetzt die feste Absicht, das Ziel in eine Handlung umzusetzen. Es 
folgt die präaktionale Vorbereitung (Phase 4), in welcher Massnahmen erarbeitet werden, die die Umsetzung auch in herausfordernden Momenten erlaubt/möglich macht. So kann letztlich die Handlung folgen (Phase 5).

Der Coachingprozesss ist Teil eines u. U. wesentlich länger andauernden Veränderungsprozesses von KlientInnen (Orlinsky et al. 2004). Anfangs- und Endpunkt des Veränderungsprozesses sind oft kaum feststellbar, da sich KlientInnen manchmal nicht mehr erinnern können, wo und wann sich der Veränderungswunsch das erste Mal manifestierte. Noch schwieriger bis unmöglich ist die zeitliche Fixierung eines Endpunktes, da Veränderungsprozesse sehr lange, manchmal auf unbestimmte Zeit, fortwirken können.

Indem das Rubikon-Modell aufzeigt, wie Veränderungsprozesse ablaufen, prädisponiert es eine Handlungslogik im Ablauf des Coachingprozesses, welche sich in viele bestehende praxisorientierte Coaching-Phasenmodelle (vgl. unten) einpflegen lässt. In unserem Ansatz wählen wir den Weg der Verknüpfung von empirisch überprüften Veränderungsmodellen mit eben solchen praxisorientierten Coaching-Phasenmodellen, weil damit der Konzeption empirisch überprüfte Modelle zu Grunde gelegt werden können und das TSPP-Modell eine zeitliche Sequenzierung der Coachingprozesse ermöglicht.

\subsection{Responsivität}

Wie erwähnt, stellt es für die Forschung eine große Herausforderung dar, die Erzeugung von Wirksamkeit und damit das Ermöglichen von Veränderung empirisch zu erfassen. Diese Schwierigkeit gründet darin, dass es sich bei Coaching und Beratung um komplexe kommunikative Wechselwirkungen zwischen Coach und KlientIn handelt, die in ihrer Ganzheit nur schwer zu erfassen und zu beschreiben sind. Stiles et al. (1998) und Kramer und Stiles (2015) umschreiben diese angesprochene Schwierigkeit mit dem Begriff der ,appropriate responsiveness“ (AR). AR bedeutet, dass TherapeutInnen, Beratende oder Coaches versuchen sollten, in jedem Moment für die anwesenden KlientInnen das zu tun, was für diese am besten ist. Was das momentan Beste ist, müssen Beratende und TherapeutInnen in Reaktion auf die KlientInnen und mit Bezugnahme auf ihr Theorie- und Handlungssystem von Moment zu Moment entlang der Sitzung entscheiden. Beratende werden somit zu einer Art MediatorIn, die zwischen KlientInnen und Theorie vermitteln. Obwohl dies für die Praxis eine äusserst anspruchsvolle Aufgabe ist, ist sie gleichzeitig doch auch der berufliche Alltag. Ein wesentlich größeres Problem stellt appropriate responsiveness für die Prozessforschung dar ,... because it undermines many conclusions based on linear reasoning and linear statistics" (Kramer und Stiles 2015, S. 277). Die Herausforderungen der AR für die Prozessforschung wird nachfolgend geklärt.

\subsection{Coaching-Prozessforschung}

Das Ziel der psychologischen Prozessforschung liegt darin, „,... durch ein besseres Verständnis der psychischen Prozesse eine effektivere Gestaltung von psychologischen Interventionen zu ermöglichen. Prozessforschung versucht, die expliziten Wirkprinzipien und Veränderungsmechanismen herauszuarbeiten“ (Lutz 2005, S. 116). Prozessforschung wird hier somit nicht als Selbstzweck verstanden, sondern als ein Mittel für die Prozess-Ergebnisforschung. Letztendlich soll es darum gehen, die Ergebnisentstehung zu erklären und Beratungsprozesse wirkungsvoller und effizienter zu gestalten. Prozessstudien fokussieren dabei z. B. allgemein die Interventionen im Coaching (Deplazes 2016). Im Kontext theoretischer Prozessüberlegungen geht es um Handlungsentscheidungen, die Coaches während des Gesprächs fällen (Geißler 2009, 2016).

An Annahmen, welche Prozesse, oder genauer, welche Wirkfaktoren zu den Ergebnissen beitragen, fehlt es nicht. Empirische Nachweise dazu sind aber noch selten. Allgemeine Wirkfaktoren haben (z.B. Greif 2008; Greif et al. 2010) und Behrendt (2006) analysiert, den Fokus auf einzelne Wirkfaktoren wie z.B. die Beziehungsgestaltung legen Ianiro und Kauffeld (2011) und Ianiro et al. (2013). Riedel (2003) entwirft eine Phänomennahe Coachingtheorie auf der Basis des erweiterten Motivationsmodells von (Heckhausen und Gollwitzer 1987) und wendet diese auf Tonband-Transkripte an; Künzli und Deplazes (2015) übernehmen im Folgenden die Methodik von Riedel (2003) und wenden sie auf ein Transkript eines E-Coachings an. Ihnen gelingt es mit diesem Vorgehen, Ergebnisse des Coachings zu erklären und zu bewerten.

Eine Kritik an der Prozessforschung in der Psychotherapie, die auf den ersten Blick paradox klingt, ist dabei unmittelbar mit dem Responsivitätsbegriff von Stiles et al. (1998) verbunden: In einer idealen Welt ist die Korrelation zwischen einzelnen Prozesskomponenten (z. B. Wirkfaktoren) und dem Ergebnis gleich Null. Dies wird von Stiles et al. (1998) wie folgt begründet: Erhalten KlientInnen exakt das Optimum einer Prozesskomponente, ist eine weitere Erhöhung oder Verminderung dieser Komponente nicht mehr hilfreich. Bewegt sich eine Prozesskomponente im Bereich des Optimums, wird das Ergebnis, falls es in einem direkten Zusammenhang mit der Prozesskomponente steht, daher konstant bleiben. Mit anderen Worten: Je responsiver die TherapeutInnen, desto geringer der Zusammenhang zwischen der Prozesskomponente und der spezifischen Ergebnisvariable. Das gleiche gilt für KlientInnenvariablen wie z.B. Alter, Geschlecht, Persönlichkeit etc. oder Settingvariablen. Ist der/die Coach optimal responsiv oder ist das Setting (z. B. Anzahl Stunden) genau auf die Bedürfnisse der KlientInnen/Coachees ausgerichtet, wird das Ergebnis für die KlientInnen maximal. Angemessene Responsivität 
glättet die Unterschiede in den KlientInnenvariablen soweit, bis der Zusammenhang zwischen Prozesskomponenten und Ergebnis verschwindet. Technisch formuliert bedeutet dies, dass die Interaktion der unabhängigen Variablen mit dem TherapeutInnenverhalten durch AR aufgefangen wird, so dass die Ergebnisvarianz Null beträgt (vgl. auch Silberschatz 1994; Stiles 1988, 1994, 1996; Stiles und Shapiro 1989, 1994).

Zu ähnlichen Aussagen kamen auch (Hill et al. 1981). Sie fanden keinen positiven Zusammenhang zwischen nonverbalem TherapeutInnenverhalten (z.B. Nicken, Lächeln) und dem Ergebnis. Die Erklärung von Hill et al. (1981) lautet: Vermutlich ist es nicht die Häufigkeit des nonverbalen Verhaltens, sondern das perfekte Timing (siehe auch die Kritik an der Untersuchung linearer Zusammenhänge von Hill et al. 1981).

Das Timing der verbalen Interaktion rückt linguistische Forschung in den Fokus. Sie arbeitet mit verschrifteten Coaching-Gesprächen, die den zeitlichen Verlauf widerspiegeln (Graf 2015, 2017a, 2018; Graf und Jautz 2019; Winkler 2017). Als ein Ergebnis dieser Forschung entwickelte Graf abduktiv das Modell der kommunikativen Basis-Aktivitäten (Graf 2015), das als Beschreibungs- und Analysemodell für Coachingprozesse verwendet werden kann. Dieses Modell wird im Anschluss vorgestellt.

\subsubsection{Das Modell der kommunikativen Basisaktivitäten nach Graf}

Dieses Modell basiert auf vier Aktivitäten: Definieren der Situation (vor allem Orientierung bezüglich des primären Zwecks und der Art und Weise der Interaktion), Gestalten der Beziehung (z. B. Etablieren der Rollen „Coach“ und „KlientIn“), Ko-Konstruieren der Veränderung (Definition und Bearbeitung des Anliegens und Ziels sowie Sicherung des Ergebnistransfers) und Evaluieren des Coachings (Evaluieren des Coachings und der Zusammenarbeit bzw. der Beziehung). Die einzelnen Aktivitäten bestehen jeweils aus wiederkehrenden kommunikativen Aufgaben, zu deren Lösung Coach und KlientIn bestimmte diskursive Praktiken und konkrete sprachliche Realisierungen zur Verfügung stehen (Graf 2015, 2019). Graf (2015) beschreibt mit ihrer Heuristik Coaching bzw. Coachingprozesse als activity type, der auf Basisaktivitäten aufbaut, die sich schleifenartig entlang einzelner Sitzungen sowie des gesamten Prozesses wiederholen (können). In ihrer Inter- und Intraaktivitätsdimension tragen die Basisaktivitäten der personen-orientierten und flexiblen Gestaltung von Coaching Rechnung, da sie Variabilität im thematischen und interaktiven Ablauf je nach Voraussetzungen, Vorerfahrungen etc. der KlientInnen ermöglichen (Graf und Jautz 2019). Trotz der Abkehr des Modells von einer strengen Linearität hält auch Graf fest, dass die Aktivitäten entlang des Prozesses eine unterschied- liche Gewichtung erfahren. So engagieren sich Coach und KlientIn zu Beginn eines Coachingprozesses in besonderem Masse in der Aktivität „Definieren der Situation“, die Aktivität ,Evaluieren des Coachings“" tritt hingegen gegen Ende einer Sitzung, bzw. eines Prozesses, verstärkt auf. Mengenmässig konnte die Aktivität „Ko-Konstruieren der Veränderung“ am häufigsten in den Transkripten gefunden werden; d.h. im gesamten Coachinggespräch bearbeiten Coach und KlientIn am meisten jene kommunikativen Aufgaben, die mit der Basisaktivität Ko-Konstruieren der Veränderung zu tun haben. Die Basisaktivität Gestalten der Beziehung wird von Coach und KlientIn im Sinne einer Daueraufgabe entlang des gesamten Prozesses bearbeitet, wobei auch hier zu Beginn eines Prozesses eine explizitere Bearbeitung zu beobachten ist.

Diese Studie ist bis dato die einzige, die sich empirisch der Gesamtstruktur von Coachingprozessen widmet und als Datenbasis authentische Coachingprozesse verwendet. Das Modell der kommunikativen Basisaktivitäten bildet somit eine zentrale Grundlage für die vorliegende Konzeption des TSPP-Modells. Die Aktivität Ko-Konstruieren der Veränderung soll jedoch für das hier zu entwickelnde Modell spezifiziert werden. Des Weiteren sollen, wie oben dargestellt, die Erkenntnisse aus empirisch überprüften Veränderungsmodellen in den Coachingprozess übertragen werden. Da mit der Studie von Graf (2015) die empirische Ausgangslage sehr beschränkt ist, soll in einem nächsten Schritt auch ein Blick auf die Coaching-Praxisliteratur geworfen werden, wo Phasenmodelle ein zentraler Bestandteil vieler Hand- und Lehrbücher sind.

\subsubsection{Phasenmodelle im Coaching: State of the Art}

Bei den Phasenmodellen der Praxisliteratur handelt es sich um rein deskriptive Modelle, die nicht empirisch überprüft wurden, teilweise jedoch präskriptive Elemente beinhalten.

Die Bedeutung, die Phasen in der Praxis zugeschrieben werden, unterscheidet sich zwischen den AutorInnen deutlich: So beschreibt beispielsweise Radatz (2003) einen ,klar strukturierten Ablauf, der von Coaching zu Coaching nur in seinem darin angewandten Instrumentarium variiert" ( $\mathrm{Ra}$ datz 2003, S. 121). Entsprechend betont sie: „Jede Phase ist so wichtig, dass auf sie keinesfalls verzichtet werden kann“ (S. 121). Schmidt (2004) geht im Gegensatz davon aus, dass sich Phasen weder starr trennen lassen, noch, dass sie zeitlich regelhaft aufeinander folgen. Letztlich sei es immer das Feedback der KlientInnen, welches Hinweise darauf gebe, in welcher Phase sich der Prozess befinde. Schmidt (2004) beschreibt die Phasen als hilfreich, für die „Ordnung der enormen Flut von Informationen“ (S. 123) und um die „Planung von Interventionen gemeinsam mit den Klienten“ (S. 123) zu entwickeln. Dieselbe Meinung vertritt Lippmann (2013) und ergänzt, dass in der Realität die Phasen 
„spiralförmig“ (S. 37-38) verlaufen. Die weiteren AutorInnen, die mit Phasenmodellen argumentieren, ordnen sich im Spektrum dieser beiden Positionen ein.

Die Bezugsgrösse der Beschreibung, also ob die Phasenmodelle eine einzelne Sitzung oder einen gesamten Coachingprozess, der sich über mehrere Sitzungen erstreckt, darstellen, wird meist nicht ausgeführt. Exemplarisch sei hier auf Lippmann (2014) verwiesen, der die Phasen im Kontext ganzer Coachingprozesse diskutiert, aber anmerkt, dass sich der Ablauf eines gesamten Prozesses mit dem Ablauf einer einzelnen Sitzung mehr oder weniger deckt, da die Phasen nicht zwingend in der beschriebenen Reihenfolge durchlaufen würden. Ebenfalls unterschiedlich fällt der Differenzierungsgrad der Phasen aus. Während z.B. Schmidt (2004) mit zehn Phasen und Radatz (2003) mit acht Phasen feingliedrige Beschreibungen gewählt haben, fassen König und Volmer (2002) oder Looss (2006) mit vier Phasen jeweils grössere Einheiten zusammen.

Vergleicht man die Phasenmodelle der gängigen Praxisliteratur, finden sich bei genauerer Betrachtung - in aller Unterschiedlichkeit - grosse Überschneidungen. Im Folgenden wird ein Überblick über die in der Praxisliteratur gefundenen Phasen gegeben:

$\mathrm{Zu}$ Beginn steht eine Orientierungsphase (König und Volmer 2002), ein Erstkontakt (Schreyögg 2012) oder Einstieg (Radatz 2003). In dieser Phase stehen das gegenseitige Kennenlernen und die Klärung von Voraussetzungen, Erwartungen und Bedürfnissen im Fokus, so dass letztlich entschieden werden kann, ob das Coaching gemeinsam weitergeführt wird.

Als ein weiterer Schritt werden die Analyse der Situation und die Zielfindung beschrieben. Lippmann (2013) und Schreyögg (2012) fassen die beiden Phasen zusammen, Rauen (2005) beschreibt für die Klärung der Ausgangssituation und Ziele jeweils eine eigene Phase und Schmidt (2004) beschreibt ausschliesslich die Entwicklung der Zielvision.

Die meisten AutorInnen beschreiben eine Phase, in welcher die Verträge und Kontrakte ausgehandelt werden. Dabei geht es einerseits um formale Bedingungen, andererseits um den psychologischen Auftrag, also um die Arbeitsbeziehung und die soziale Basis auf der das Coaching durchgeführt werden kann. Diese Kontraktphase wird von den AutorInnen an unterschiedlicher Stelle beschrieben. Lippmann (2013) und Schmidt (2004) beispielsweise schliessen diese der ersten Orientierungsphase direkt an. Radatz (2003) und Schreyögg (2012) bearbeiten die Kontrakte erst im Anschluss an die Situationsanalyse sowie die Zielverhandlungen.

Des Weiteren folgt eine Phase des eigentlichen Coachings (Schreyögg 2012), die auch Intervention (Loebbert 2013; Rauen 2005) oder Veränderungsphase (König und
Volmer 2002) genannt wird. Diese Phase wird von anderen AutorInnen in bis zu fünf Phasen unterteilt (Schmidt 2004).

Es folgt der Abschluss des Coachings. Dieser beinhaltet meist die Auswertung und den Abschluss, wobei die Reihenfolge hier variieren kann. Es geht um eine Rückschau auf den Prozess, eine persönliche Evaluation und darum, die gemeinsame Arbeit zu würdigen (Schmidt 2004).

Auf der Basis der beschriebenen Theorien, Modelle und Ansätze wird in dem nun folgenden zweiten Teil des Beitrags das TSPP-Modell konzipiert. Einschränkend ist anzumerken, dass der Anspruch des Modells im Moment rein deskriptiver, einordnender Natur ist. Die Verbindung der einzelnen Modellelemente muss noch offen bleiben.

\section{Konzeption des Turn-Sequenz-Phase- Prozess Modells (TSPP-Modell)}

Der Coachingprozess vollzieht sich in und durch die Gespräche zwischen Coach und KlientIn. Ein Modell, das für die Strukturierung von auf Gesprächen aufbauenden Coachingprozessen entwickelt wird, kann aus unterschiedlich differenzierten Komponenten bestehen. Es ist somit eine zentrale Aufgabe vor der Entwicklung des Modells die Aufteilung des Prozesses in Komponenten zu reflektieren. Wie dargestellt, werden zur Beschreibung von Coachingprozessen in der vorhandenen Literatur primär Phasen zu Hilfe genommen: sowohl empirische Veränderungsmodelle als auch deskriptive Modelle zum Coachingprozess aus der Praxis strukturieren die Prozesse in mehr oder weniger differenzierte Phasen.

Phasen folgen einer sequentiellen Handlungslogik und beinhalten Aufgaben, die Coach und KlientInnen im Rahmen der jeweiligen Phase gemeinsam lösen. Während des Bearbeitens der entsprechenden Aufgabe kann es aber zu Rückgriffen oder Vorgriffen auf andere Phasen im Prozess kommen. Als Beispiel sei genannt, dass es nach der Zieldefinition zu einer Erkenntnis kommt und im Gespräch bemerkt wird, dass das Ziel somit präzisiert oder verändert werden soll. In diesem Moment müssen Coach und KlientIn in die Phase der Zieldefinition zurückkehren. Es ist jedoch auch möglich, dass während des Bearbeitens der Aufgabe einer Phase nur ein kurzer Exkurs im Sinne einer „side sequence“ (Jefferson 1972) oder ,insertion sequence“" (Schegloff 1972) auf eine andere Phase gemacht wird (z. B. „Da fällt mir gerade noch ein ...“). Bei diesen und anderen Fällen stellt sich die Frage, wie mit solchen Phänomenen umgegangen werden kann und ob und wie jede einzelne Sequenz von Coach oder KlientIn einer Phase zugeordnet werden kann bzw. muss.

Um gerade auch solche Phänomene beobachten, beschreiben und analysieren zu können, scheint es hilfreich, eine feinere Einheit zur Strukturierung der Coachingpro- 
Abb. 1 TSPP-Modell

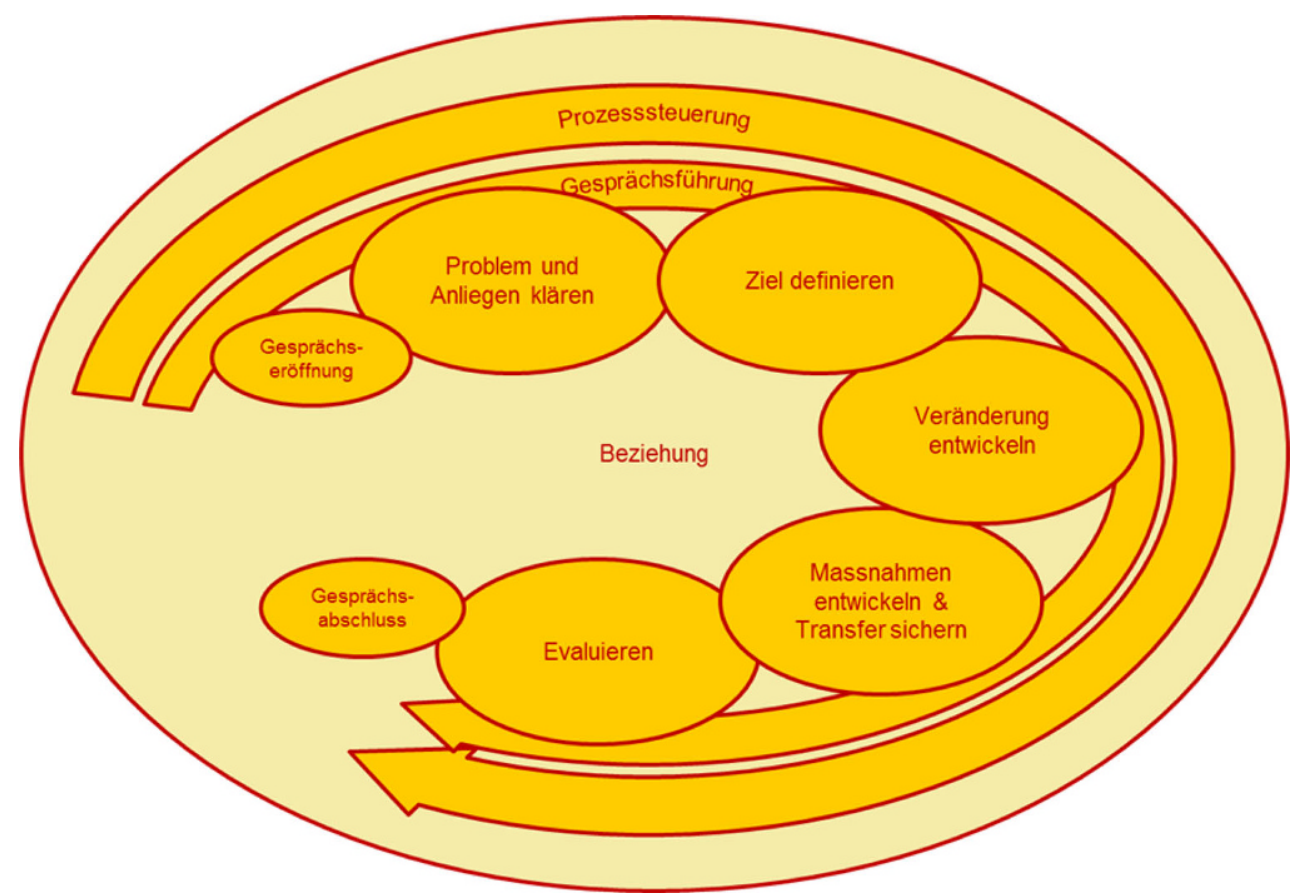

zesse zu definieren. Das folgende Modell (vgl. Abb. 2) umfasst drei Ebenen: Turn, Sequenz, Phase. Sequenzen setzen sich aus mehreren Turns und Phasen aus mehreren Sequenzen zusammen. Obwohl die Prozessinhalte, aufbauend auf Turns, Sequenzen und Phasen, von Coach und KlientIn gemeinsam gestaltet werden, liegt die Steuerung des Prozesses in der Verantwortung der Coaches. Coaches führen den Prozess ihrer Theorie und Handlungslogik folgend von Phase zu Phase. Die konkrete Umsetzung der Prozessteuerung und damit das Initiieren, Bearbeiten und Abschließen der Phasen erfolgt in der Gesprächsführung. Als Basis für das Coachinggespräch wird die Arbeitsbeziehung zwischen Coach und KlientIn betrachtet, welche im Modell als „Grund“ (im Sinne von Boden) dargestellt ist.

Nachfolgend wird das Modell in zwei Abbildungen dargestellt. Dies ermöglicht es sowohl den Überblick über die Phasen (vgl. Abb. 1) als auch deren Präzisierung in Sequenzen und Turns (vgl. Abb. 2) verständlich darzustellen. Einleitend werden die zu Grunde liegenden back-stage Aktivitäten im Sinne Goffmans (1959) vorgestellt: die Arbeitsbeziehung, die Prozesssteuerung und die Gesprächsführung. Es folgen die Kernkomponenten „Turn“, „Sequenz“ und „Phase“. Die Kernkomponente der Phase wird auch inhaltlich in ihren Realisierungsformen beschrieben: „Problem und Anliegen klären“, „Ziel definieren“, ,,Veränderung entwickeln“, „Massnahmen entwickeln und Transfer sichern“ und „Evaluieren“.

\subsection{Die Back-stage Aktivitäten Arbeitsbeziehung, Prozesssteuerung und Gesprächsführung}

\subsubsection{Beziehung}

$\mathrm{Zu}$ Beginn jedes Coachings muss ein „Feld für die Kooperation geschaffen werden" (Schmidt 2004, S. 124). Wenn davon ausgegangen wird, dass im Coaching mit autonomen und sich selbst organisierenden Personen gearbeitet wird, setzt dies voraus, dass Coaches dem Erleben von KlientInnen wertschätzend und akzeptierend begegnen. Dieses würdigende Verhalten der Coaches ermöglicht es den KlientInnen, kooperativ und aktiv in Interaktion zu treten. In der Praxis wird oft der Begriff des „Pacing“ dafür verwendet. Coaches lassen sich würdigend auf das Erleben der KlientInnen ein, ohne ihre eigenen Bedürfnisse und Bedingungen aus den Augen zu lassen (Schmidt 2016). Dieses Pacen und die Pflege der Arbeitsbeziehung beginnt bei der ersten Begegnung und bleibt im gesamten weiteren Cochingprozess eine Aufgabe. Berninger-Schäfer (2018) beschreibt die Beziehung - sowohl im face-to-face Coaching als auch im Online-Coaching - als ,phasenunabhängiges, durchgehendes Basisverhalten“ (BerningerSchäfer 2018, S. 78) und stellt sie auch visuell als dem Phasenverlauf parallel liegend dar. D.h., dass die Beziehung stets und gleichzeitig zu allen Phasen gestaltet wird.

Aus gesprächsanalytischer Sicht sprechen Graf und Spranz-Fogasy (2018a) bei Beziehungsgestaltung von einer „Kernaufgabe“ helfender Interaktionen wie Coaching und Graf (2015) definiert das Gestalten der Beziehung 
Abb. 2 Präzisierung der Phasen im TSPP-Modell

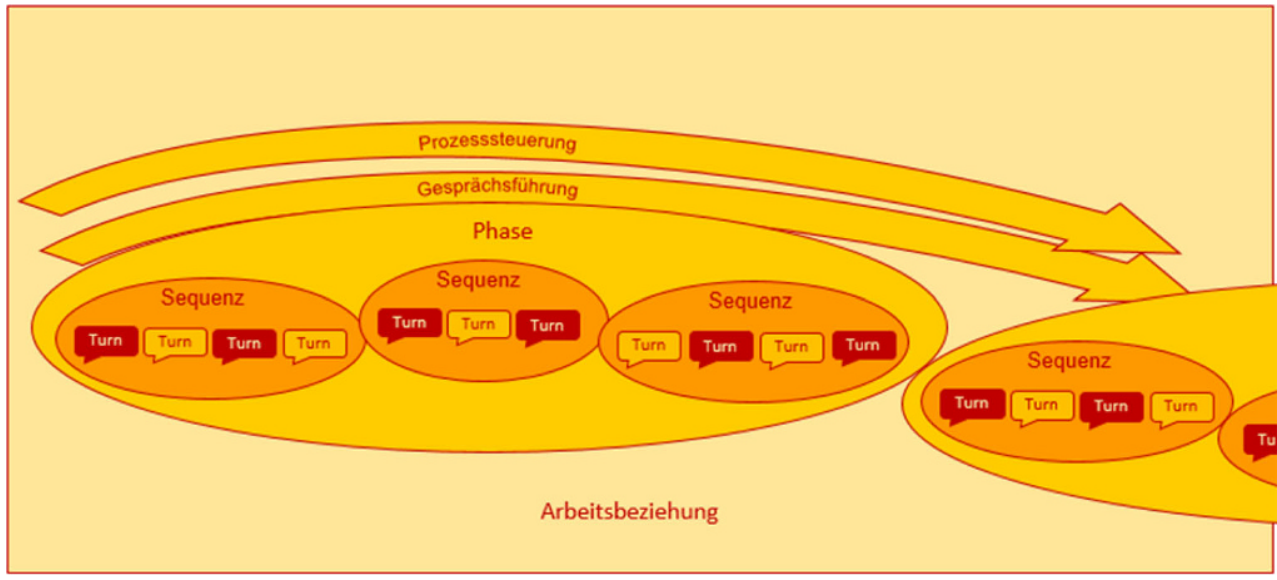

als eine der vier Basisaktivitäten. Grawe (1994) fasst verschiedenen Prozess-Outcome-Studien zusammen und hält fest, dass eine gute Arbeitsbeziehung „eine absolut notwendige Voraussetzung für ein gutes Therapieergebnis“ (S. 8) ist. Die Arbeitsbeziehung kann demnach als „basso continuo“ (Seliger 2006) des Coachings betrachtet werden. Deshalb wurde sie im Modell auch nicht als Prozesskomponente, sondern als Basis der gesamten Zusammenarbeit dargestellt.

\subsubsection{Prozesssteuerung}

Prozessberatung, im Unterschied zur Expertenberatung, weist die Verantwortung für den Inhalt den KlientInnen, die für den Prozess den Coaches zu (Schein 2003; Schreyögg 2012). Damit wird der Prozess besonders inhaltlich gemeinsam von Coach und KlientIn gestaltet. Die Steuerung desselben liegt in der Hand des Coaches. Coaches sind z. B. dafür verantwortlich, dass gemeinsam ein Ziel vereinbart wird und dass das Coaching-Gespräch bzw. die CoachingGespräche eines Prozesses darauf ausgerichtet sind. Dabei orientieren sich Coaches in Tempo, Rhythmus, Ablauf und den zu besprechenden Themen (mehr oder weniger) an den KlientInnen. Die Phasen folgen einer Handlungslogik, doch wird diese entsprechend dem Verlauf des Coachings und den Bedürfnissen der KlientIn individuell angepasst (vgl. Graf und Jautz 2019). Somit orientiert sich der Coach in der Prozesssteuerung im Sinne der appropriate responsiveness (Kramer und Stiles 2015) sowohl an den KlientInnen als auch an den Phasen des Coachingprozesses. So soll bspw. die Sinnhaftigkeit des gemeinsam definierten Ziels im Laufe der Beratung überprüft werden und es ist durchaus möglich, dass es sich im Verlaufe des Prozesses verändert. In diesem Fall muss die Phase erneut durchlaufen werden und es muss gemeinsam ein neues Ziel für den weiteren Verlauf des Coachings definiert werden. Entsprechend muss der weitere Verlauf des Coachings angepasst werden.
Die konkrete Umsetzung dieser Prozesssteuerung durch die Coaches zeigt sich in der Gesprächsführung.

\subsubsection{Gesprächsführung}

Die Gesprächsführung realisiert die konkrete Umsetzung des Coachings im Gespräch. Winkler (2017) verortet die Gesprächssteuerung in Anlehnung an Tiittula (2001) durch den Coach auf den Ebenen der Gesprächsorganisation (z. B. Initiierung und Beendigung des Coachinggesprächs oder Abgrenzung von rahmendem Small-Talk), der Themenkonstitution (z.B. fokussiert der/die Coach auf bestimmte thematische Aspekte in der Erzählung der KlientIn, während andere Aspekte nicht aufgegriffen werden) sowie der Handlungskonstitution (z.B. dienen bestimmte Frage-AntwortSequenzen der Bearbeitung des Anliegens auf der intrapersonalen Ebene, während andere Frage-Anwort-Sequenzen der Diagnose des Problems dienen) und beschreibt sie als entscheidendes Kriterium einer gelingenden Gesprächsführung. Die Gesprächsführung von Coaches ist dabei geprägt von professionellen Agenden, die sich im Bearbeiten konkreter Aufgaben im Kontext der einzelnen Phasen zeigen. Diese Agenden prädeterminieren mehr oder weniger grob den Gesprächsverlauf in seiner internen Aufgabenund Handlungslogik, d.h. die Abfolge und Ausgestaltung der Phasen. Die Einhaltung der Agenden liegt in der Macht der Coaches. Die Gesprächsorganisation erfolgt in grossem Masse durch Orientierungshandlungen des Coachs (Winkler 2017). Diese entsprechen in Teilen Grafs (2015) kommunikativen Praktiken im Kontext der Basisaktivität „Definieren der Situation“. Sie umfassen die Metakommunikation (Graf 2017b) mit welcher der Coach KlientInnen über die Art und Weise der Interaktion informiert und das weitere Vorgehen gemeinsam bestimmt wird. Darüber hinaus lenken Coaches den Verlauf des Gesprächs, in dem sie z.B. regeln, wer das Rederecht erhält, worüber gesprochen wird und welche Handlungen die GesprächspartnerInnen durchzuführen haben (Tiittula 2001). D.h. mit Blick auf die Pha- 
sen wird durch die Coaches z. B. gesteuert, wann eine Phase initiiert wird, wie sie durchgearbeitet wird und wann und wie sie beendet wird.

\subsection{Die Kernkomponenten des TSPP-Modells - Turn, Sequenz, Phase}

Wie einleitend dargestellt, strukturiert das Modell den Coachingprozess zeitlich und handlungslogisch in Turns, Sequenzen und Phasen (vgl. Abb. 2). Diese Kernkomponenten werden nachfolgend vorgestellt.

Als kleinste Einheit des Modells wird der Turn (=Redebeiträge) gewählt. Ein Turn beschreibt das, was Coach oder KlientIn zwischen zwei Sprecherwechseln sagt, d.h. während er oder sie an der Reihe ist (Goffmann 1974, S. 201), „we take turns to talk“ (Drew 2013, S. 131). Turns sind von unterschiedlicher Länge und können sich über nur minimale (Rück-)Meldungen als auch über lange Ausführungen erstrecken; Turns bauen sich aus unterschiedlichen turnconstructional units auf. Obwohl jeder Turn für sich steht, ist er immer notwendigerweise Teil einer Gesprächssequenz und hat eine bestimmte Position innerhalb dieser Sequenz. Aufgrund des Organisationsprinzips der Sequenzialität von Gesprächen (Auer 1999; Sacks et al. 1974; Schegloff 2007; Stivers 2013) sind die einzelnen Turns dabei durch das Prinzip der konditionalen Relevanz miteinander verbunden: so bedingt eine Frage als nächsten Redebeitrag eine bestimmte Art der Erwiderung, eine Antwort bzw. Response (Graf und Spranz-Fogasy 2018b). Dabei werden Turns von den GesprächsteilnehmerInnen selbst als Frage etc. interpretiert: ,... wir erkennen aus der Art und Weise, wie der andere seine nächsten Handlungen formuliert, wie er unsere erste Handlung verstanden hat" (Auer 1999, S. 138).

Sequenzen bilden die nächste, mittlere, Analyseeinheit unseres Modells. Sequenzen bestehen aus mehreren Turns, die aufgrund des Prinzips der konditionalen Relevanz bzw. der bedingten Erwartbarkeit sowohl thematisch als auch gesprächsorganisatorisch miteinander verbunden sind (Henne und Rehbock 2001): ,sequence of turns are not haphazard but have a shape or structure, and can be tracked for where they came from, what is being done through them, and where they might be going“"(Schegloff 2007, S. 3). Ein Turn verweist innerhalb einer Sequenz dabei immer auf den Vorherigen und bildet die Voraussetzung für den nachfolgenden Turn: „Initiierende Aspekte projizieren relevante Folgeaktivitäten, respondierende Aspekte verweisen zurück; die erstgenannten sind kontextdeterminierend, die letztgenannten kontextdeterminiert" (Tiittula 2001, S. 1362). Auch Sequenzen sind von unterschiedlicher Länge; so realisieren adjacency pairs oder Gesprächsschrittpaare wie Gruß - Gegengruß minimale Sequenzen (Stivers 2013), allerdings beinhalten Sequenzen zumeist mehr als zwei aufeinanderfolgende Turns (vgl. Graf und Spranz-Fogasy 2018b;
Schegloff 2007). Durch den (auch) thematischen Zusammenhang von Sequenzen lassen sich mit Hilfe des Modells auch sogenannte side sequences (Jefferson 1972) bzw. insertion sequences (Schegloff 1972) isolieren, die nicht unmittelbar dem Bearbeiten der aktuellen Aufgabe im Rahmen der jeweiligen Phase dienen.

Die nächst höhere Strukturierungsebene des TSPP-Modells bildet die Phase, die aus mehreren Sequenzen besteht. Die Ebene der Phasen bezieht sich auf die übergeordnete strukturelle Organisation von Coaching-Gesprächen (Robinson 2013). Spiegel und Spranz-Fogasy (2001) zufolge wird der Begriff der Phase in der Literatur als komplexe Einheiten innerhalb von Gesprächen bezeichnet, die wesentliche Handlungs- oder Themenkomplexe ausmachen, eine interaktionslogische Reihenfolge implizieren und intern differenziert sind. Je nach Gesprächstyp gibt es dabei spezifische Verlaufsformen von Gesprächen und damit spezifische Gesprächsphasen, wobei jedes Gespräch die komplementären, relativ einfach strukturierten Phasen der Gesprächseröffnung und der Gesprächsbeendigung sowie eine sehr komplexe Gesprächsmitte enthält (Brinker und Sager 2010; Spiegel und Spranz-Fogasy 2001). Während sich die Phase der Gesprächsmitte auch bei alltäglichen Gesprächen als die Kernphase darstellt, ist sie dort weder thematisch noch gesprächsorganisatorisch a priori in feste Bestandteile strukturiert. Anders bei professionellen Gesprächen wie etwa Coaching, wo in der Kernphase die gemäß der professionellen Agenda zu bearbeitenden Aufgaben im Zentrum stehen. Die Gliederung in Phasen ermöglicht den Entwurf eines vorläufigen Bildes vom Gesamtverlauf, auch wenn die konkrete Entwicklung letztlich nicht prognostizierbar ist (Schiersmann und Thiel 2012). Für Coaching geht dieses Modell (in Anlehnung an empirisch überprüfte Veränderungsmodelle und Basisaktivitäten sowie Phasenmodellen aus der Praxis, vgl. oben) von den Aufgaben „Problem und Anliegen klären“, „Ziel definieren“, „Veränderung entwickeln“, „Maßnahmen entwickeln \& Transfer sichern“ und „Evaluieren“ aus. Allerdings werden Phasen hier primär als thematische und handlungsorientierte Einheit eher im Sinne von Aktivitäten (Graf 2015) verstanden, da „die Modelle einer linearen und schrittweise prozessualen Abfolge von Gesprächsphasen nicht der Gesprächsrealität entsprechen“ (Spiegel und Spranz-Fogasy 2001, S. 17).

\subsection{Die Phasen des TSPP-Modells}

Im Folgenden werden die Phasen des Modells beschrieben. Vorab die Gesprächseröffnung, gefolgt von den fünf Phasen, die die Kernphasen des Coaching-Gesprächs bilden, sowie der Gesprächsabschluss. Die Tatsache, dass das TSPP-Modell Gesprächseröffnung und Gesprächsabschluss als Elemente des Modells berücksichtigt und im Folgenden beschreibt, trägt dem Modell zugrunde liegenden Ver- 
ständnis von Coaching als Gespräch Rechnung. In diesem Verständnis fungiert besonders die Gesprächseröffnung als wichtige interaktive, thematische und relationale Vorbereitung für den weiteren Gesprächs- bzw. Coaching-Verlauf (Gebel und Speck 1991; Menz et al. 2008; Nowak 2010).

\subsubsection{Gesprächseröffnung'}

In der Gesprächseröffnung leisten Coach und KlientIn verbal, z.B. durch Grußformeln, und nonverbal, z.B. durch Blickkontakt, die wechselseitige Identifizierung und stellen eine gemeinsame Gesprächsbereitschaft her; beides ist Bestandteil der Situationsdefinition, die die Beteiligten gemeinsam aushandeln. Darüber hinaus leistet die Gesprächseröffnung Orientierung, da sich die ProtagonistInnen (zumeist) noch nicht kennen und auf beiden Seiten eine gewisse Unsicherheit besteht. Hier ist es die Aufgabe von Coaches, Verantwortung für das Gespräch zu übernehmen und zu versuchen Sicherheit zu geben, z. B. mittels Smalltalk (Pick et al. in Vorbereitung; Spranz-Fogasy 2005). Gleichzeitig beginnt die gemeinsame Aushandlung von Handlungsaufgaben und Gesprächsthemen; dies markiert den Übergang zur nächsten Phase. Seliger (2006) vergleicht hierbei den Beginn der Begegnung zwischen Coach und KlientIn mit einem Musikstück: in „einer Symphonie oder einer Oper werden in der Overtüre alle wesentlichen Themen angespielt, die sich in der Folge weiter entfalten werden“" (Seliger 2006, S. 8). Die Dauer der Gesprächseröffnung kann stark variieren je nach Bekanntheitsgrad der Teilnehmenden und deren Zeitbudget, der Öffentlichkeit oder Privatheit der Gesprächssituation, die wievielte Sitzung ein Coachinggespräch ist etc. Grundsätzlich wird bei der Gesprächseröffnung von Coaching-Gesprächen als professionellem Gespräch, im Unterschied zu Alltagsgesprächen, ein relativ rasches Zur-Sache-Kommen erwartet (Spiegel und Spranz-Fogasy 2001).

\subsubsection{Problem und Anliegen klären}

Die Prozess-Komponente umfasst das Eruieren, wie die Idee der Beratung entstanden ist, welche Erwartungen die KlientInnen mitbringen, welche Erklärungen zu den Problemzusammenhängen bestehen, welche Vorstellungen von

\footnotetext{
${ }^{1}$ Hierbei muss unterschieden werden, ob es sich um die Gesprächseröffnung der ersten Coaching-Sitzung oder der weiterer Sitzungen handelt. Während die Gesprächseröffnung der ersten Sitzung ,wechselseitig unbekannte Personen in unbekannte-aber-kontextuell-identifizierbare Personen transformiert" (Reitemeier 1994, S. 232 f.), können Coach und KlientIn in den Gesprächseröffnungen der folgenden Sitzung auf dieses Wissen bereits zurückgreifen. Eine gemeinsame Aushandlung von Handlungsaufgaben und Gesprächsthemen sowie die (Wieder-)Herstellung der Beziehung sind aber auch in allen Folge-Eröffnungen kommunikativ zentral.
}

einer gewünschten Lösung bestehen und welche Beteiligten wie betroffen sind.

Im Rubikon-Modells wird zwischen dem Bedürfnis und dem Motiv (Storch und Krause 2007) unterschieden. Der Schritt von einem Bedürfnis hin zu einem bewussten Motiv wird in der Phase „Problem und Anliegen klären“ erörtert. Es wird davon ausgegangen, dass KlientInnen sich vor jeder Veränderung klarwerden müssen, welche Wünsche und Anliegen sie überhaupt in Tat umsetzen möchten. Dazu wird die Wünschbarkeit und Realisierbarkeit gegeneinander abgewogen. Zum Abwägen gehört auch die Reflexion über mögliche unmittelbare und langfristige Folgen, die mit einer Veränderung einhergehen können (Achtziger und Gollwitzer 2010). Mit anderen Worten benennt Graf (2015) dieselbe Aufgabe als „Diagnostizieren“ im Rahmen der Basisaktivität Ko-Konstruieren von Veränderung. Sie umfasst ebenfalls das Explorieren und Definieren des Anliegens.

Konkret beginnen Coach und KlientIn nach der Gesprächseröffnung in dieser Phase ihre gemeinsame Arbeit am Anliegen. Dabei begegnen Coaches den Klärungsversuchen der KlientInnen (idealer Weise) mit einem würdigenden Pacing (Schmidt 2004). Weiter ist es die Aufgabe der Coaches, zu versuchen die Komplexität des Anliegens zu reduzieren und mindestens eine vorläufige Strukturierung dessen vorzunehmen, um eine möglichst anschauliche Rekonstruktion des Ist-Zustandes zu erarbeiten (Schreyögg 2012). Die Klärung des Anliegens und des Problems ermöglicht es nicht nur den Coaches das Problem zu verstehen, sondern erweitert auch die Perspektive der KlientInnen und hilft diesen sich darüber klar zu werden, was sie im Coaching konkret bearbeiten möchten. Diese Erkenntnis ermöglicht die Definition des Ziels, was als die darauf folgende Phase beschrieben wird.

\subsubsection{Ziel definieren}

Diese Phase umfasst den Gesprächsabschnitt, in welchem Coach und KlientIn das Ziel besprechen und definieren. Im Rubikon-Modell wird keine Phase der Zieldefinition beschrieben. Es wird jedoch davon ausgegangen, dass der „Rubikon überschritten wird“, wenn aus den Wünschen und Anliegen der KlientInnen eine Zielintention wird und damit das Commitment zur Veränderung entsteht (Achtziger und Gollwitzer 2010). Da dieser Moment dem Modell gar den Namen verleiht, muss von einem zentralen Moment im Veränderungsprozess ausgegangen werden. Im Unterschied zu einem intrapsychischen Veränderungsmodell handelt es sich beim Coachingprozess um die Interaktion zwischen Coach und KlientIn. Für die Zusammenarbeit ist es unumgänglich, dass sich beide ProtagonistInnen an dem Ziel orientieren können, weshalb dieses gemeinsam besprochen und definiert werden muss. Es wird diskutiert, dass 
die Wahrscheinlichkeit ein Ziel zu erreichen steigt, wenn KlientInnen die Ziele selbst formulieren. Doch liegt es in der Verantwortung der Coaches, dass ein Ziel formuliert wird und dieses dem Setting des Coachings entspricht bzw. angemessen ist.

Demnach soll das Ziel auf jeden Fall explizit benannt werden, oft hilft es, dieses gar zu notieren. Das heisst, auch wenn es sich bei der Zieldefinition um einen zeitlich oft kürzeren Gesprächsabschnitt handelt, stellt sie für den Beratungsprozess ein zentraler Moment dar, der durchaus als Dreh- und Angelpunkt der Beratung benannt werden kann. Die Prozessteuerung orientiert sich an der Zieldefinition.

\subsubsection{Veränderung entwickeln}

In der folgenden Prozess-Komponente bzw. Phase begleiten also Coaches die KlientInnen entlang des definierten Ziels um Veränderungen zu entwickeln. KlientInnen befinden sich in der Sprache des Rubikon-Modells in der präaktionalen Phase und erarbeiten Strategien um die festgelegten Ziele zu realisieren. Von Schreyögg (2012) wird diese Phase ,eigentliches Coaching“ (S. 362) genannt. Im Modell von Graf (2015) handelt es sich hierbei um die kommunikative Aufgabe des Intervenierens im Kontext der Basisaktivität Ko-Konstruieren von Veränderung.

Diese Phase umfasst im Kern das Bearbeiten des Anliegens auf der intra- und der interpersonalen Ebene. Die Vorgehensweise kann vielfältig sein und unterscheidet sich je nach theoretischer Ausrichtung des Coachings. Es können z. B. bisherige Lösungsversuche besprochen und Ressourcen aus der Vergangenheit aktiviert werden, indem überlegt wird, wann schon einmal ähnliche Herausforderungen gemeistert werden konnten. Es können Ausnahmen im Problemerleben erarbeitet, analysiert und für die Problemlösung beigezogen werden. Letztlich werden die Lösungsmöglichkeiten und deren Wirkung auch im Kontext des betroffenen sozialen Systems überprüft.

Konnte eine aus der Perspektive der KlientInnen sinnvolle Veränderung entwickelt werden, können in einem nächsten Schritt konkrete Handlungsmassnahmen entwickelt werden und damit der Transfer gesichert werden.

\subsubsection{Massnahmen entwickeln und Transfer sichern}

Neben dem Entwickeln von Strategien umfasst die präaktionale Phase des Rubikon-Modells auch das Schmieden von Handlungsplänen. Je konkreter ein Bild von einer neuen Zukunft gezeichnet wird, desto eher kann dieses auch umgesetzt werden. Demnach werden im Coaching konkrete Massnahmenpläne erarbeitet oder z. B. anstehende Gespräche in Rollenspielen geübt. Neben dem Entwickeln von Transfer-Praktiken beschreibt Graf (2015) das Verankern von Erlerntem, welches in späteren Momenten des Coachings bearbeitet werden soll.

Ein Coaching muss nicht zwingend die Veränderung von Verhalten oder Handlungsmustern zum Ziel haben. Ebenso ist es möglich, die Veränderung von Deutungsmustern oder eine Perspektivenerweiterung zu verfolgen. Entsprechend muss die Massnahmenplanung nicht zwingend auf der Handlungsebene erfolgen. Wenn das Ziel die Veränderung von Deutungsmuster ist, könnte z. B. erarbeitet werden, wie zukünftig in Momenten von Zweifeln oder Rückfällen in alte Denk- oder Verhaltensmuster vorgegangen werden kann (vgl. hierzu die linguistische Therapieforschung, die neben der Veränderung von Handlungsmustern die Bedeutung der Veränderung von Denk- bzw. Deutungsmustern hervorhebt (für einen Überblick siehe Scarvaglieri in Vorbereitung)).

\subsubsection{Evaluation}

In den Worten des Rubikon-Modells (Achtziger und Gollwitzer 2010) geht es in der postaktionalen Evaluation um den Vergleich der Soll- mit der Ist-Situation der Umsetzung der Ziele. Die Tatsache, dass Graf (2015) abduktiv diese Aktivität definiert, zeigt, dass das Evaluieren im CoachingGeschehen eine relevante Grösse zu sein scheint.

Vor dem Abschluss des Coachings, aber auch am Ende der einzelnen Sitzungen wird im Hinblick auf das definierte Ziel und die dazugehörigen Kriterien ein Blick zurück auf den Prozess geworfen. In der Praxis geschieht dies z. B. durch Skalenfragen. Gegebenenfalls können weitere Handlungen definiert werden oder gar die Zielintention adaptiert werden. Ebenfalls wird oftmals die Arbeitsatmosphäre bzw. die Zusammenarbeit zwischen Coach und KlientIn angesprochen und evaluiert. Dies spiegelt die besondere Bedeutung der Beziehung in helfenden Berufen wie Coaching wider.

\subsubsection{Gesprächsabschluss ${ }^{2}$}

Der Abschluss des Gesprächs ist die zur Gesprächseröffnung komplementäre Gesprächsphase: Die Beendigung eines Gesprächs wird eingeleitet, wenn zwischen den InteraktantInnen darüber Einverständnis erzielt worden ist, dass das „eigentliche“ Gespräch, d.h. die Behandlung der Gesprächsthemen, abgeschlossen ist (Brinker und Sager 2006, S. 104). Eine gemeinsame Themenbeendigung z. B. im Kontext der Phase „Evaluation“ geht als Abschluss der Kernphase des Gesprächs der eigentlichen Beendigungsphase voraus. Hier erfolgen die Würdigung der Interaktion,

\footnotetext{
${ }^{2}$ In Analogie zur Gesprächseröffnung muss bei der Gesprächsbeendigung unterschieden werden, ob es sich um den Abschluss der ersten Sitzung, einer weiteren Sitzung oder um die Beendigung des gesamten Coachingprozesses handelt.
} 
oftmals mittels einer (gegenseitigen) Danksequenz sowie einer Wunschsequenz und die eigentliche Verabschiedung. Darüber hinaus sind Terminabsprachen oftmals Bestandteil der Abschlussphase (Schegloff und Sacks 1973; Graf 2015).

\section{Diskussion}

Der vorliegende Artikel bietet eine theoretische Auseinandersetzung mit existierenden Modellen und Theorien aus verschiedenen Traditionen der Coaching-Forschung und entwickelt darauf aufbauend ein neues Modell, das TSPPModell (Turn-Sequenz-Phase-Prozess-Modell). Dieses soll als Strukturierungshilfe der Coaching-Prozessforschung dienen, die mit folgender Herausforderung zu kämpfen hat: Zum momentanen Zeitpunkt können wir empirisch nachweisen, dass Coaching wirkt, aber noch nicht erklären, wie diese Wirkung entsteht. Die Schwierigkeit diese Wirkung zu erklären gründet darin, dass es sich bei Coaching um komplexe kommunikative Wechselwirkungen zwischen Coach und KlientIn handelt, die in ihrer Gesamtheit nur schwer fassbar sind. Gleichzeitig kann diese Fragestellung als eine zentrale Forschungslücke definiert werden.

Die Strukturierung mittels des TSPP-Modells orientiert sich an zwei etablierten Wissensständen zum Coaching: 1) Coaching realisiert sich im Gespräch, welches auf Turns und Sequenzen aufbaut. 2) Ziel eines jeden Coachings ist Veränderung; Veränderung lässt sich in Phasen beschreiben. Die Organisation in Turns und Sequenzen erlaubt es den Beteiligten, Aufgaben im Coaching so zu bearbeiten, dass Veränderung in den KlientInnen entwickelt werden kann. Die zu bearbeitenden Aufgaben orientieren sich an den Phasen der Veränderung; das Coachinggespräch entwickelt sich somit entlang dieser Phasen, welche inhaltlich über ihren Beitrag zu Veränderung definiert werden.

Durch diese Integration der Verlaufsperspektive und der Inhaltsperspektive von Coachingprozessen betritt das TSPP-Modell theoretisches und methodisches Neuland und kann einen wertvollen Beitrag zum Phänomen der appropriate responsiveness leisten. Gleichzeitig ist jedes Modell, auch das vorliegende, eine vereinfachte Abbildung der sehr viel komplexeren Realität. Eine kritische und kontinuierliche Weiterarbeit, vor allem im Kontext der genauen Verbindung der Modellelemente, ist notwendig. Darüber hinaus muss das TSPP-Modell in einem nächsten Schritt empirisch überprüft werden.

Open Access Dieser Artikel wird unter der Creative Commons Namensnennung 4.0 International Lizenz (http://creativecommons.org/ licenses/by/4.0/deed.de) veröffentlicht, welche die Nutzung, Vervielfältigung, Bearbeitung, Verbreitung und Wiedergabe in jeglichem Medium und Format erlaubt, sofern Sie den/die ursprünglichen Autor(en) und die Quelle ordnungsgemäß nennen, einen Link zur Creative Commons Lizenz beifügen und angeben, ob Änderungen vorgenommen wurden.

\section{Literatur}

Achtziger, A., \& Gollwitzer, P.M. (2010). Motivation und Volition im Handlungsverlauf. In Motivation und Handeln (4. Aufl. S. 309-335). Berlin: Springer.

Auer, P. (1999). Sprachliche Interaktion: Eine Einführung anhand von 22 Klassikern (1. Aufl.). Tübingen: De Gruyter.

Behrendt, D.P.P. (2006). Wirkung und Wirkfaktoren von psychodramatischem Coaching - Eine experimentelle Evaluationsstudie. Zeitschrift für Psychodrama und Soziometrie, 5(1), 59-87.

Berninger-Schäfer, E. (2018). Online-coaching. Wiesbaden: Springer.

Brinker, P.D. K., \& Sager, P.D.S.F. (2010). Linguistische Gesprächsanalyse: Eine Einführung (5. Aufl.). Berlin: Erich Schmidt.

Burt, D., \& Talati, Z. (2017). The unsolved value of executive coaching: a meta-analysis of outcomes using randomised control trial studies. International Journal of Evidence Based Coaching and Mentoring, 15(2), 17-24.

Deplazes, S. (2016). KaSyCo - Kategoriensysteme zur Analyse von Coachingprozessen

Deppermann, A. (2008). Gespräche analysieren - Eine Einführung. Wiesbaden: VS.

Deppermann, A. (2018). Konversationsanalyse und diskursive Psychologie. In Handbuch Qualitaitve Forschung in der Psychologie (S. 643-661). Wiesbaden: VS.

Drew, P. (2013). Turn design. In J. Sidnell \& T. Stivers (Hrsg.), The handbook of conversation analysis (S. 131-149). Oxford: WileyBlackwell.

Fillery-Travis, A., \& Cox, E. (2014). Researching coaching. In E. Cox, T. Bachkirova \& D. Clutterbuck (Hrsg.), The complete handbook of coaching (S. 445-459). London: SAGE.

Gebel, S., \& Speck, A. (1991). Untersuchung der Eröffnung von Therapiegesprächen. In D. Flader (Hrsg.), Verbale Interaktion. Studien zur Empirie und Methodologie der Pragmatik (S. 19-26). Stuttgart: Metzler.

Geißler, H. (2009). Die inhaltsanalytische „Vermessung“ von Coachingprozessen. In Coachingwissen. Denn sie wissen nicht, was sie tun (S. 93-125).

Geißler, H. (2016). Die Grammatik des Coachens: Eine empirische Rekonstruktion. Wiesbaden: Springer.

Goffman, E. (1959). The presentation of self in everyday life. New York: Anchor Books.

Goffmann, E. (1974). Frame analysis. New York: Northeastern University Press.

Graf, E.-M. (2015). Kommunikative Basisaktivitäten im CoachingGespräch: Ein linguistischer Beitrag zur Coaching-Prozessforschung. Coaching Theorie \& Praxis, 1(1), 5-14. https://doi.org/ 10.1365/s40896-015-0001-x.

Graf, E.-M. (2017a). Management Coaching - Ein arbeitsweltliches Beratungsgespräch zwischen Selbst-Reflexion und Output Orientierung? In I. Pick (Hrsg.), Beraten ist Sprechen. Eine linguistische Typologie zu Beratungsgesprächen in verschiedenen Handlungsfeldern (S. 77-95). Bern: Peter Lang.

Graf, E.-M. (2017b). Forms and functions of metadiscourse in goaloriented talk-in-interaction: the case of executive coaching. In A. Onysko, E.-M. Graf, W. Delanoy, G. Sigott \& N. Dobrić (Hrsg.), The polyphony of English studies: a Festschrift for Allan James (S. 113-132). Tübingen: Gunter Narr.

Graf, E.-M. (2018). Äußerung für Äußerung zum Coaching-Prozess: Das Potential einer gesprächsanalytisch fundierten Coaching-Prozessforschung. In R. Wegener, A. Fritze, M. Hänseler \& M. Loebbert (Hrsg.), Coaching-Prozessforschung. Forschung und Praxis im Dialog (S. 141-161). Göttingen: Vandenhoeck \& Ruprecht.

Graf, E.-M. (2019). The Pragmatics of Executive Coaching. Amsterdam: John Benjamins.

Graf, E.-M., \& Spranz-Fogasy, T. (2018a). Helfende Berufe - Helfende Interaktionen. In K. Birkner \& N. Janich (Hrsg.), Handbuch Text und Gespräch (S. 418-442). Mouton: De Gruyter Mouton. 
Graf, E.-M., \& Spranz-Fogasy, T. (2018b). Welche Frage, wann und warum? - Eine qualitativ-linguistische Programmatik zur Erforschung von Frage-Sequenzen als zentrale Veränderungspraktik im Coaching. Coaching Theorie \& Praxis. https://doi.org/10.1365/ s40896-018-0021-4.

Graf, E.-M., \& Jautz, S. (under review). „Und wie, wie entsteht Veränderung. Und damit haben wir ja auch mit dir zu tun“ - Einblicke in die inter- und intra-aktivitätsspezifische Dimension der kommunikativen Basisaktivität „Veränderung ko-konstruieren“ im Führungskräfte-Coaching. In E.-M. Graf, C. Scarvaglieri \& T. Spranz-Fogasy (Hrsg.), Pragmatik der Veränderung. Problemund lösungsorientierte Kommunikation in helfenden Berufen (Band ,Studien zur Pragmatik“). Tübingen: Gunter Narr.

Grawe, K. (1994). Psychotherapie ohne Grenzen. Von den Therapieschulen zur Allgemeinen Psychotherapie. Verhaltenstherapie und psychosoziale Praxis, 26(3), 357-370.

Grawe, K. (1998). Psychologische Therapie. Göttingen: Hogrefe.

Greif, S. (2008). Coaching und ergebnisorientierte Selbstreflexion. Theorie, Forschung und Praxis des Einzel- und Gruppencoachings. Göttingen: Hogrefe.

Greif, S., \& Benning-Rohnke, E. (2015). Konsequente Umsetzung von Zielen durch Coaching: Praktisch nützliche Erkenntnisse aus der Grundlagenforschung und ihre Anwendung. Coaching Theorie \& Praxis, 1(1), 25-35. https://doi.org/10.1365/s40896-015-0003-8.

Greif, S., Schmidt, F., \& Thamm, A. (2010). The rating of eight coaching success factors-oberservation manual

de Haan, E., Bertie, C., Day, A., \& Sills, C. (2010). Critical moments of clients and coaches: a direct-comparison study. International Coaching Psychology Review, 5, 109-128.

Heckhausen, H., \& Gollwitzer, P.M. (1987). Thought contents and cognitive-functioning in motivational versus volitional states of mind. Motivation and Emotion, 11(2), 101-120.

Heckhausen, J., \& Heckhausen, H. (Hrsg.). (2010). Motivation und Handeln. Bd. 4. Berlin: Springer.

Henne, H., \& Rehbock, H. (2001). Einführung in die Gesprächsanalyse. Berlin: De Gruyter.

Hill, C.E., Siegelman, L., Gronsky, B., Sturniolo, F., \& Fretz, B. R. (1981). Nonverbal communication and counseling outcome. Journal of Counseling Psychology, 28(3), 203-212.

Ianiro, P. M., \& Kauffeld, S. (2011). Black-Box Coaching-Prozess: Beziehungsaufbau und -gestaltung auf Grundlage interpersonaler Basisdimensionen. LOCCS-Symposium, LMU München. München: LMU.

Ianiro, P. M., Schermuly, C.C., \& Kauffeld, S. (2013). Why interpersonal dominance and affiliation matter: an interaction analysis of the coach-client relationship. Coaching: An international Journal of Theory, Research and Practice, 6(1), 25-46. https://doi.org/10. 1080/17521882.2012.740489.

Jefferson, G. (1972). Side sequences. In D. Sudnow (Hrsg.), Studies in social interaction (S. 294-338). New York: Free Press.

Jones, R. J., Woods, S. A., \& Guillaume, Y.R.F. (2016). The effectiveness of workplace coaching: a meta-analysis of learning and performance outcomes from coaching. Journal of Occupational \& Organizational Psychology, 89(2), 249-277. https://doi.org/10. 1111/joop.12119.

König, E., \& Volmer, G. (2002). Systemisches Coaching. Handbuch für Führungskräfte, Berater und Trainer. Weinheim: Beltz.

Kramer, U., \& Stiles, W.B. (2015). The responsiveness problem in psychotherapy: a review of proposed solutions. Clinical Psychology: Science and Practice, 22(3), 277-295. https://doi.org/10. 1111/cpsp.12107.

Künzli, H., \& Deplazes, S. (2015). Die Veränderung subjektiver Theorien. In H. Geißler \& R. Wegener (Hrsg.), Bewertung von Coachingprozessen (S. 81-97). Wiesbaden: Springer. https://doi.org/ 10.1007/978-3-658-04140-3 4.

Lippmann, E. (2013). Coaching. Angewandte Psychologie für die Beratungspraxis (3. Aufl.). Berlin, Heidelberg: Springer.
Lippmann, E. (2014). Phasen eines gesamten Coachingprozesses. In A. Ryba, D. Pauw, D. Ginati \& S. Rietmann (Hrsg.), Professionell coachen (S. 364-369). Weinheim: Beltz.

Loebbert, M. (2013). Professional Coaching. Stuttgart: Schäffer-Poeschel.

Looss, W. (2006). Unter vier Augen: Coaching für Manager. Bergisch Gladbach: EHP.

Lutz, W. (2005). Verlaufs- und Prozessforschung. In F. Petermann \& H. Reinecker (Hrsg.), Handbuch der Klinischen Psychologie und Psychotherapie (S. 112-121). Göttingen: Hogrefe.

Menz, F., Lalouschek, J., \& Gstettner, A. (2008). Effiziente ärztliche Gesprächsführung. Wien: LIT.

De Meuse, K.P., Dai, G., \& Lee, R. J. (2009). Evaluating the effectiveness of executive coaching: beyond ROI? Coaching: An international Journal of Theory, Research and Practice, 2, 117-134. https://doi.org/10.1080/17521880902882413.

Nowak, P. (2010). Eine Systematik der Arzt-Patient-Interaktion. Frankfurt: Peter Lang.

Orlinsky, D.E., Ronnestad, M.H., \& Willutzki, U. (2004). Fifty years of psychotherapy process-outcome research: continuity an change. In M. J. Lambert (Hrsg.), Bergin and Garfield's handbook of psychotherapy and behavior change (S. 307-389). New York: Wiley.

Passmore, J., Peterson, D., \& Freire, T. (2013). The psychology of coaching and mentoring. In J. Passmore, D. Peterson \& T. Freire (Hrsg.), The Wiley-Blackwell handbook of the psychology of coaching and Mentoring (S. 1-11). Chinchester: Wiley-Blackwell.

Pick, I., Graf, E.-M. \& Aksu, Y. (in Vorb.). Gesprächseröffnungen in Coaching, Supervision und Rechtsberatung: Ein diskursanalytischer Vergleich der Themen- und Handlungskomplexe sowie der Eröffnungsinitiativen.

Prochaska, J.O., Velicer, W.F., Guadagnoli, E., Rossi, J. S., \& DiClemente, C.C. (1991). Patterns of change-dynamic typology applied to smoking cessation. Multivariate Behavioral Research, 26(1), 83-107.

Radatz, S. (2003). Beratung ohne Ratschlag. Systemisches Coaching für Führungskräfte und BeraterInnen. Wien: Verlag systemisches Management.

Ramseyer, F., \& Tschacher, W. (2011). Nonverbal synchrony in psychotherapy: coordinated body movement reflects relationship quality and outcome. Journal of Consulting and Clinical Psychology, 79(3), 284-295. https://doi.org/10.1037/a0023419.

Ramseyer, F., \& Tschacher, W. (2014). Nonverbal synchrony of headand body-movement in psychotherapy: different signals have different associations with outcome. Frontiers in Psychology. https:// doi.org/10.3389/fpsyg.2014.00979.

Rauen, C. (2005). Der Ablauf eines Coaching-Prozesses. In Handbuch Coaching (3. Aufl. S. 273-288). Göttingen: Hogrefe.

Reitemeier, U. (1994). Beraten und institutioneller Kontext. In W. Nothdurft, U. Reitemeier \& P. Schröder (Hrsg.), Beratungsgespräche. Analyse asymmetrischer Dialoge (S. 230-259). Tübingen: Narr.

Riedel, J. (2003). Coaching für Führungskräfte: Erklärungsmodell und Fallstudien. Wiesbaden: Deutscher Universitätsverlag.

Robinson, J. (2013). Overall structural organization. In J. Sidnell \& T. Stivers (Hrsg.), The handbook of conversation analysis (S. 257-280). Oxford: Wiley-Blackwell.

Sacks, H., \& Schegloff, E. A. (1973) Opening up closings, Semiotica, $8(4), 289-327$.

Sacks, H., Schegloff, E. A., \& Jefferson, G. (1974). A simplest systematics for the organization of turn-taking for conversation. Language, 50(4), 696. https://doi.org/10.2307/412243.

Scarvaglieri, C. (under review). Veränderung durch Verstehen in der Psychotherapie. In E.-M. Graf, Scarvaglieri, C. \& T. Spranz-Fogasy (Hrsg.), Pragmatik der Veränderung. Problem- und lösungsorientierte Kommunikation in helfenden Berufen. (Band „Studien zur Pragmatik“). Tübingen: Gunter Narr. 
Schegloff, E. A. (1972). Sequencing in conversational openings. In J. Gumperz \& D. Hymes (Hrsg.), Directions in sociolinguistics (S. 346-380). New York: Holt, Rinehart \& Winston.

Schegloff, E. A. (2007). A primer in conversation analysis. Sequence organization in interaction, Bd. 1. Cambridge: Cambridge University Press.

Schein, E.H. (2003). Prozessberatung für die Organisation der Zukunft. Bergisch Gladbach: EHP.

Schiersmann, Ch., \& Thiel, H.-U. (2012). Beratung als Förderung von Selbstorganisationsprozessen - eine Theorie jenseits von „Schulen“ und „Formaten“. In C. Schiersmann \& H.-U. Thiel (Hrsg.), Beratung als Förderung von Selbstorganisationsprozessen (S. 14-78). Göttingen: Vandenhoeck \& Ruprecht.

Schmidt, G. (2004). Liebesaffären zwischen Problem und Lösung. Heidelberg: Auer.

Schmidt, G. (2016). Einführung in die hypnosystemische Therapie und Beratung (Compact) (7. Aufl.). Heidelberg: Carl-Auer Verlag $\mathrm{GmbH}$.

Schreyögg, A. (2012). Coaching. Frankfurt: Campus.

Schwarzer, R. (2008). Modeling health behavior change: how to predict and modify the adoption and maintenance of health behaviors. Applied Psychology, 57(1), 1-29. https://doi.org/10.1111/j. 1464-0597.2007.00325.x.

Seliger, R. (2006). Erste Begegnung in der Beratung (Vorabdruck). In Systemische Organisationsentwicklung und Beratung bei Veränderungsprozessen in Organisationen. Ein Handbuch. Heidelberg: Carl Auer Verlag.

Silberschatz, G. (1994). Abuse and disabuse of the drug metaphor in psychotherapy research: Hold on to the baby as you throw out the bath. Journal of Consulting and Clinical Psychology, 62(5), 949-951. https://doi.org/10.1037//0022-006X.62.5.949.

Sonesh, S. C., Coultas, C. W., Lacerenza, C.N., Marlow, S.L., Benishek, L. E., \& Salas, E. (2015). The power of coaching: a meta-analytic investigation. Coaching: An International Journal of Theory, Research and Practice, 8(2), 73-95. https://doi.org/10.1080/ 17521882.2015.1071418.

Spiegel, C., \& Spranz-Fogasy, T. (2001). Aufbau und Abfolge von Gesprächsphasen. In K. Brinker, G. Antos, W. Heinemann \& S. Sager (Hrsg.), In Text- und Gesprächslinguistik. Ein internationales Handbuch zeitgenössischer Forschung (Bd. 2, S. 1241-1251). Berlin: De Gruyter Mouton.

Spranz-Fogasy, T. (2005). Kommunikatives Handeln in ärztlichen Gesprächen - Gesprächseröffnung und Beschwerdenexploration. In M. Neises, S. Dietz \& T. Spranz-Fogasy (Hrsg.), Psychosomatische Gesprächsführung in der Frauenheilkunde. Ein interdiszipli- närer Ansatz, zur verbalen Interaktion. Stuttgart: Wissenschaftliche Verlagsgesellschaft.

Stiles, W. B. (1988). Psychotherapy process-outcome correlations may be misleading. Psychotherapy: Theory, Research, Practice, Training, 25(1), 27-35. https://doi.org/10.1037/h0085320.

Stiles, W.B. (1994). Drugs, recipes, babies, bathwater, and psychotherapy process-outcome relations. Journal of Consulting and Clinical Psychology, 62(5), 955-959. https://doi.org/10.1037/ 0022-006X.62.5.955.

Stiles, W. B. (1996). When more of a good thing is better: a reply to hayes et al. Journal of Consulting and Clinical Psychology, 64(5), 915-918. https://doi.org/10.1037/0022-006X.64.5.915.

Stiles, W.B., \& Shapiro, D.A. (1989). Abuse of the drug metaphor in psychotherapy process-outcome research (Special Issue: Psychotherapy Process Research). Clinical Psychology Review, 9(4), 521-543. https://doi.org/10.1016/0272-7358(89)90007-X.

Stiles, W. B., \& Shapiro, D. A. (1994). Disabuse of the drug metaphor: psychotherapy process-outcome correlations. Journal of Consulting and Clinical Psychology, 62(5), 942-948. https://doi.org/10. 1037/0022-006X.62.5.942.

Stiles, W. B., Honos-Webb, L., \& Surko, M. (1998). Responsiveness in psychotherapy. Clinical Psychology: Science and Practice, 5(4), 439-458. https://doi.org/10.1111/j.1468-2850.1998.tb00166.x.

Stivers, T. (2013). Sequence organization. In J. Sidnell \& T. Stivers (Hrsg.), The handbook of conversation analysis (S. 191-209). Oxford: Wiley-Blackwell.

Storch, M., \& Krause, F. (2007). Selbstmanagement - ressourcenorientiert. Grundlagen und Trainingsmanual für die Arbeit mit dem Zürcher Ressourcen Modell. Bern: Huber.

Theeboom, T., Beersma, B., \& van Vianen, A.E. (2014). Does coaching work? A meta-analysis on the effects of coaching on individual level outcomes in an organizational context. The Journal of Positive Psychology, 9(1), 1-18. https://doi.org/10.1080/ 17439760.2013.837499.

Tiittula, L. (2001). Formen der Gesprächssteuerung. In Ein internationales Handbuch zeitgenössischer Forschung (Bd. 2, S. 1361-1374). Berlin: De Gruyter.

Whitworth, L., Kimsey-House, K., Kimsey-House, H., \& Sandahl, P. (1998). Co-active coaching: new skills for coaching people toward success in work and life. Palo Alto: Davis-Black Publishing.

Winkler, O. (2017). Anwendung der Gesprächsanalyse als FeedbackInstrument im Chat-Coaching. Coaching Theorie \& Praxis, 3(1), 17-27. https://doi.org/10.1365/s40896-017-0016-6. 